\title{
Glasslike Arrest in Spinodal Decomposition as a Route to Colloidal Gelation
}

\author{
S. Manley, ${ }^{1, *}$ H. M. Wyss, ${ }^{1}$ K. Miyazaki, ${ }^{2, \dagger}$ J. C. Conrad, ${ }^{1}$ V. Trappe,${ }^{3}$ L. J. Kaufman,${ }^{2, \dagger}$ \\ D. R. Reichman, ${ }^{2, \dagger}$ and D. A. Weitz ${ }^{1}$ \\ ${ }^{1}$ Department of Physics \& DEAS, Harvard University, Cambridge, Massachusetts 02138, USA \\ ${ }^{2}$ Department of Chemistry \& Chemical Biology, Harvard University, Cambridge, Massachusetts 02138, USA \\ ${ }^{3}$ Department of Physics, University of Fribourg, CH-1700 Fribourg, Switzerland
}

(Received 14 December 2004; published 1 December 2005)

\begin{abstract}
Colloid-polymer mixtures can undergo spinodal decomposition into colloid-rich and colloid-poor regions. Gelation results when interconnected colloid-rich regions solidify. We show that this occurs when these regions undergo a glass transition, leading to dynamic arrest of the spinodal decomposition. The characteristic length scale of the gel decreases with increasing quench depth, and the nonergodicity parameter exhibits a pronounced dependence on scattering vector. Mode coupling theory gives a good description of the dynamics, provided we use the full static structure as input.
\end{abstract}

Colloid-polymer mixtures exhibit a rich phase behavior. Depletion of polymer between two colloidal particles induces an attractive interaction whose magnitude $U$ is set by the polymer concentration in the free volume, $c_{p}$. The range of this interparticle attraction is determined by the radius of the polymer $R_{p}$ and is expressed by $\xi=R_{p} / a$, with $a$ the particle radius. The phase behavior is controlled by both $c_{p}$ and $\xi$, as well as the colloid volume fraction, $\phi$. The particles can form equilibrium phases, including crystals, fluids, and gases [1,2]. Additionally, there are a wide variety of nonequilibrium states, characterized by the dynamic arrest of the colloidal particles [3-6]. In the absence of polymer, crowding drives the system into a repulsive glassy state at $\phi \sim 0.58$, where particles are caged by their nearest neighbors. This dynamic arrest is captured by mode coupling theory (MCT), a mean-field-like theory which uses the static structure factor, $S(q)$, to predict the dynamics $[7,8]$. Upon addition of a small amount of polymer, arrest is no longer driven by crowding, but instead by weak attraction between particles; surprisingly, this attractive glass can also be described by MCT [4,9]. In both cases, the dominant length scale in the structure corresponds to the nearest-neighbor particle separation. By contrast, at lower $\phi$ and higher $U$, the system can gel, forming a space-spanning network characterized by an additional, larger length scale. For systems at very low $\phi$ and sufficiently high $U$, gelation is a consequence of kinetics; fractal clusters grow and ultimately form a gel by clustercluster aggregation $[10,11]$. The theory for kinetic aggregation naturally includes the larger length scale, which is the cluster size. By contrast, at higher $\phi$ and lower $U$, phase separation, such as spinodal decomposition, can drive large-scale structuring, influencing the pathways for gel formation [12-14]. In this case, the mechanisms for arrest during gelation are still unclear. Gelation requires the formation of a solidified, space-spanning network. In spinodal decomposition this is driven by dynamic arrest within the connected colloid-rich region; this may result from percolation [15-19], pinning [20], or a glasslike transition $[6,12,13,17,21,22]$. An experimental investigation that distinguishes these possible mechanisms, and that determines the correct underlying physics is essential to provide guidance for a theoretical description of this class of gelation.

In this Letter, we show that when gelation of colloidpolymer mixtures follows spinodal decomposition, solidification occurs via a glass transition within the colloid-rich phase. We calculate the dynamics with MCT [12], and include modes at both high and low wave vectors $q$, using as input the structure factor $S(q)$ determined by static light scattering (SLS). We find good agreement between calculated nonergodicity factors, and those measured by dynamic light scattering (DLS).

We use sterically stabilized poly-methylmethacrylate spheres, with radius $a=136 \pm 5 \mathrm{~nm}$, as determined by DLS. Particles are suspended at a volume fraction of $\phi=0.25$ in an index- and density-matching solvent mixture of cycloheptyl bromide and decalin. This allows us to probe the samples using both light scattering and microscopy, while minimizing the influence of gravity. Since the colloids are slightly charged in these solvents, samples are prepared with $1 \mathrm{mM}$ tetra-butylammonium chloride (TBAC) [23] to suppress any Coulombic interactions. Attractive depletion interactions are induced by adding linear polystyrene with a molecular weight $M_{w}=2 \times 10^{6}$ dalton. To approximate the interaction between two particles separated by a distance $r$, we use the Asakura-Oosawa potential, $\frac{U}{k_{B} T}=\Pi\left(c_{p}\right) V(r)$, where $\Pi\left(c_{p}\right)$ is the osmotic pressure, and $V(r)$ is the overlap volume of their depletion zones $[24,25]$. We determine $\Pi\left(c_{p}\right)$ by integrating the osmotic modulus, $d \Pi\left(c_{p}\right) / d c_{p}$, obtained with SLS from polystyrene solutions [26]. In all cases, $c_{p}$ is below the overlap concentration. Because we use a good solvent for polystyrene, $R_{p}$ decreases with increasing $c_{p}$; thus $\xi$ decreases from $\sim 0.3$ to $\sim 0.2$. 
We study the structures formed at different $U$ by measuring the SLS intensity, $I(q)$, over a wide range of scattering vectors. Using a goniometer with an Ar-ion laser, with a wavelength in vacuo $\lambda=514.5 \mathrm{~nm}$, we measure $I(q)$ for $2.4 \mu \mathrm{m}^{-1}<q<21 \mu \mathrm{m}^{-1}$. We use the PercusYevick (PY) [27] approximation to calculate the static structure factor for hard spheres, $S_{\mathrm{HS}}(q)$. We normalize the scattering intensity obtained from a polymer-free sample by $S_{\mathrm{HS}}(q)$ to determine the particle form factor $F(q)$ which we use to obtain $S(q)$ for all other samples. All data are affected by multiple scattering which increases the intensity near the minimum of the form factor [28], and this effect is mitigated by the normalization. To access lower $q, 0.2 \mu \mathrm{m}^{-1}<q<1.7 \mu \mathrm{m}^{-1}$, the scattered light obtained using a collimated He-Ne laser, with $\lambda=$ $633 \mathrm{~nm}$, is projected onto a screen, which is imaged using a CCD camera. The intensity is averaged around rings of constant $q$, and normalized by a factor chosen to best match the low- and high- $q$ data for the sample with $c_{p}=0$. This combination of measurements allows us to probe structures from length scales just below the particle size, $q a \sim 4$, up to tens of particle diameters, $q a \sim 0.03$.

For weak attractions, $U \leq 2.6 k_{B} T$, the dominant feature in $S(q)$ is the nearest-neighbor peak, as shown in Fig. 1. The peak position shifts to higher $q$ relative to that of $S_{\mathrm{HS}}(q)$; this reflects that particles are, on average, closer together. Concomitantly, there is a slight increase in $S(q)$ at low $q$ by comparison to $S_{\mathrm{HS}}(q)$. As we increase the attraction further, $U \leq 2.9 k_{B} T$, the magnitude of the particleparticle peak increases sharply, and its width becomes narrower, reflecting a strong increase in correlations between neighboring particles. This increase is highlighted by $\Delta S_{m}$, the difference between the value of $S(q)$ at the nearest-neighbor peak and its value at the minimum at lower $q$, shown in the inset of Fig. 1. Concurrently, an intense peak appears at small wave vectors, reflecting the sudden appearance of large-scale structures. This peak

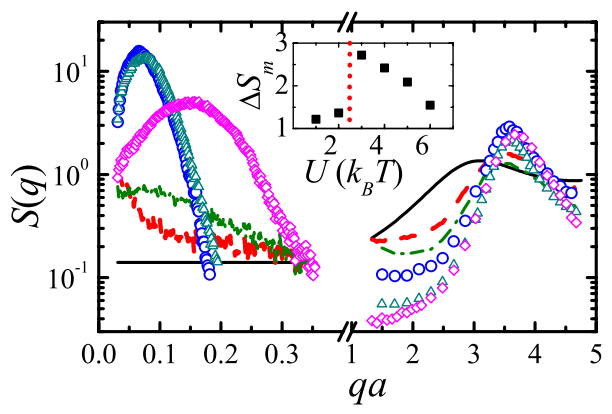

FIG. 1 (color online). Wave vector dependence of the static structure factor for $U / k_{B} T=2.46$ (dashed line), 2.62 (dasheddot line), $2.89(\bigcirc), 3.06(\triangle)$, and $3.85(\diamond)$. The solid line corresponds to the hard-sphere $S(q)$ expected at $\phi=0.25$. Inset: $U$ dependence of $\Delta S_{m}$, defined as the difference between the value of $S(q)$ at the nearest-neighbor peak and that at the minimum at $q a \sim 1$. The dotted line denotes the point at which the particle-particle position becomes highly correlated. A sharp peak appears concurrently at low $q$. appears almost immediately after mixing ceases, and shows only weak time dependence over $30 \mathrm{~min}$, shifting to slightly lower $q$. The position of the low- $q$ peak shifts to higher $q$ as $U$ is increased, indicating that the characteristic length of the system becomes smaller; this is accompanied by a reduction in the degree of spatial correlation at the nearest-neighbor length scale, indicated by a decrease in $\Delta S_{m}$. Once the gels form, we see little evidence of aging in either their structure or dynamics.

To further elucidate the morphology of the network, we use coherent anti-Stokes Raman scattering (CARS) microscopy $[29,30]$ to directly image the samples. At low interaction energies, $U \leq 2.6 k_{B} T$, there is no resolvable structure in the CARS images. However, upon a slight increase in the attraction, $U \geq 2.9 k_{B} T$, large, spacespanning networks appear, filling the entire field of view, as shown in Fig. 2. The structures are static over the $\sim 30$ min measurement time; thus, these images provide direct evidence of the presence of a dynamically arrested network that spans space. As $U$ increases, the characteristic length scale of the network decreases. This is qualitatively consistent with the $U$ dependence of the low- $q$ peak in $S(q)$; furthermore, spatial Fourier transforms of the images exhibit a peak at $q_{c}$ consistent with that measured with light scattering. The resultant characteristic sizes, $R_{c} \sim$ $\pi / q_{c}$, are shown by the circles in Fig. 2. These structures are much larger than expected for diffusion-limited cluster aggregation [10], which predicts $R_{c} \sim 3 a$, too small to be resolved in the CARS images.

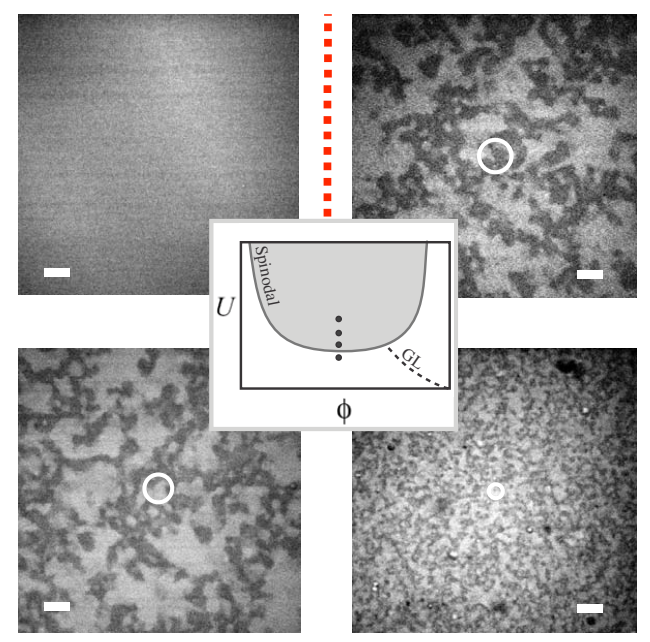

FIG. 2 (color online). Images obtained by CARS microscopy: $U / k_{B} T=2.46$ (top left); $U / k_{B} T=2.89$ (top right); $U / k_{B} T=$ 3.06 (bottom left); $U / k_{B} T=3.85$ (bottom right). The dotted line between the two upper images denotes the boundary beyond which long-lasting, space-filling structures are observed. The scale bars correspond to $10 \mu \mathrm{m}$ and the circles to $\pi / q_{c}$, where $q_{c}$ is obtained from the small angle SLS data. Inset: Schematic phase diagram for colloid-polymer mixtures as polymer concentration and colloid volume fraction are varied, where the glass transition line is marked (GL). Points denote the approximate location of our samples. 
The abrupt appearance of space-spanning networks with increasing $U$ is accompanied by a pronounced change in the dynamic structure factor, $f(q, t)$. For $U \leq 2.6 k_{B} T$, $f(q, t)$ is ergodic, as shown by the lines in Fig. 3(a), where we plot $f(q, t)$ for $q a=3.5$ as a function of the viscositycorrected delay time $t \eta_{0} / \eta$, with $\eta_{0}$ and $\eta$ the viscosities of the solvent and the background polymer solutions, respectively. The decays are reasonably well described by single exponentials and the decay times increase only slightly with $U$. For $U \geq 2.9 k_{B} T, f(q, t)$ no longer fully decays, as shown by the symbols in Fig. 3(a); this is consistent with dynamic arrest of the network over the time scales of the experiment. For these samples, we use the ensemble-averaged scattering intensity to correct the measured time-averaged data and to determine $f(q, t)[31]$.

The sudden onset of dynamically arrested networks with increasing $U$ suggests that the system crosses the spinodal line, as shown schematically in the inset of Fig. 2. Samples quenched above the spinodal line separate into interconnected colloid-poor and colloid-rich regions which span space. Gelation occurs when the local particle concentration in the colloid-rich regions is sufficiently high that the particles become arrested; this leads to a solidification of the network and arrest of the spinodal decomposition. In spinodal decomposition, the rate of increase of the local $\phi$ increases with quench depth, which depends on $U$; thus, dynamic arrest occurs more quickly for higher $U$, reducing the coarsening time of the phase separating system. Likewise, the initial characteristic length scale in spinodal decomposition decreases with quench depth. Thus, the characteristic length scale of the network decreases with increasing $U$, as shown in Figs. 1 and 2.

There are several mechanisms which may be responsible for the dynamic arrest within the colloid-rich region, including percolation [15-19], a cluster glass transition $[6,22]$, or an attractive glass transition $[12,13,17,21]$. To help elucidate the mechanism for the arrest, we measure $f(q, t)$ as a function of $q$. Although the samples remain nonergodic for all $q$, a slow decay persists even at the longest times measured, as shown for $U=2.89 k_{B} T$ in Fig. 3(b). Thus, we determine the nonergodicity factors $f_{c}(q)$ from the value of $f(q, t)$ at $t \eta_{0} / \eta=0.2 \mathrm{sec}$. For each $U, f_{c}(q)$ is not monotonic in $q$; there are pronounced increases both at low $q$ and at the nearest-neighbor peak $q a \sim 3.5$ as shown by the symbols in Fig. 4; this is reminiscent of the shape of $S(q)$. These results cannot be explained by a percolation scenario, which predicts that $f_{c}(q)$ decreases monotonically with $q$, and grows continuously from zero as $U$ increases $[18,19]$. Instead, we compare the data with the behavior of $f(q, t)$ calculated from MCT. Although MCT cannot rigorously be applied to highly heterogeneous, nonequilibrium systems [32], it should nevertheless qualitatively capture the nature of this gelation since the colloid-rich phase is solely responsible for the observed slow dynamics and dynamical arrest, and since there is a separation of time scales between local, glassy freezing and large-scale restructuring of the gel

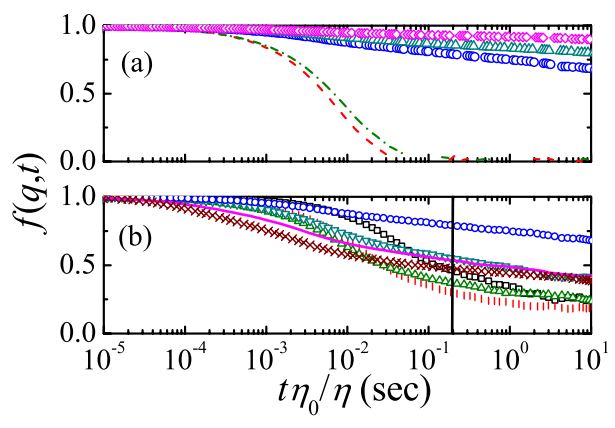

FIG. 3 (color online). (a) Dynamic structure factor at the $q$ value of the nearest-neighbor peak for $U / k_{B} T=2.46$ (dashed line), 2.62 (dashed-dot line), $2.89(\bigcirc), 3.06(\triangle)$, and $3.85(\diamond)$. To account for the increased viscosity due to the added polymer, the time axis is normalized by the ratio of the viscosity of the polymer solution to that of the solvent, $\eta / \eta_{0}$. (b) Dynamic structure factor for $U / k_{B} T=2.89$ obtained at $q a=1.29(\square)$, 1.9 (vertical bars), $2.49(\triangle), 3.03(\bigcirc), 3.52(\nabla), 4.07$ (solid line), and $4.31(\times)$. The vertical line denotes the $f(q, t)$ values used to approximate the nonergodicity factor $f_{c}(q)$.

[12]. However, to account for gelation, we must use the full $S(q)$ as input, including the peak at low $q$ which accounts for the larger length scale structures of the gel; thus, we use the measured $S(q)$ shown in Fig. 1. In addition, since the MCT results are sensitive to very high $q$ contributions of $S(q)$, we extend the data using values calculated with the Asakura-Oosawa potential [33]. The volume fraction of the colloid-rich region $\tilde{\phi}$ is left as a free parameter [34], and is chosen to best match the calculated $f(q, t)$ to experimental data. For $U=3.85 k_{B} T$, we find $\tilde{\phi}$ is within the range for which MCT predicts local nonergodicity for a homogeneous system. For $U=2.89 k_{B} T$ and $U=3.06 k_{B} T$, we find the values of $\tilde{\phi}$ to be slightly below the values required for local nonergodicity. We evaluate $f_{c}(q)$ by choosing a time for which the calculated value matches that of the experiment at $q a=3.5$. For all $U$, our MCT predictions are in excellent agreement with the data, as shown by the solid lines in Fig. 4; moreover, this agreement persists if we evaluate $f_{c}(q)$ at longer times.

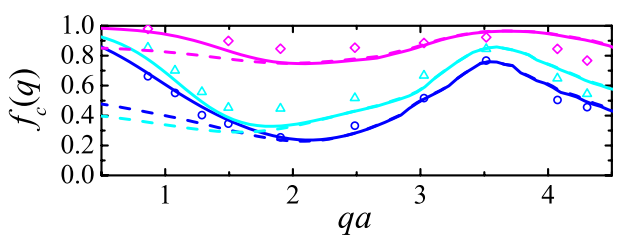

FIG. 4 (color online). Wave vector dependence of the nonergodicity factor. The symbols are the measured $f_{c}(q)$. From bottom to top the data are for $U / k_{B} T=2.89,3.06$, and 3.85. The solid lines are obtained from the MCT calculation using $S(q)$ as input; experimental $S(q)$ at low and high $q$ were smoothed and joined with a spline fit, while at the highest $q$ we use an asymptotic expression for the numerical solution of the PY equation for the Asakura-Oosawa potential, adjusted to match the data. We use values $\tilde{\phi}=0.37,0.52$, and 0.50 respectively; the dashed lines are obtained by neglecting the low angle peak. 
This agreement is remarkable considering the wide separation between network and nearest-neighbor length scales, and the highly heterogeneous structures. It highlights the critical role of glassy dynamics in the kinetic arrest of the spinodal decomposition, leading to formation of a solid network [13].

This MCT calculation captures the increase of the nonergodic parameter at low wave vectors, indicative of the presence of the peak in $S(q)$ at $q a<0.2$ [32]. This increase reflects the constraints on motion at these length scales due to the existence of the interconnected space-spanning network. If the 1ow- $q$ peak is not included, the predicted $f_{c}(q)$ is significantly lower, as shown by the dashed lines in Fig. 4. However, we find that the transition is still predicted to occur at nearly the same local volume fraction when the low- $q$ peak is excluded. This implies that the large-lengthscale structures do not drive the arrest as predicted for a cluster glass transition [12,22]; instead, gelation is driven by local arrest of the dynamics in a preformed network. To further test for a possible arrest due to the formation of a glass of clusters each comprising many particles, we recalculate the MCT using as input only the low- $q$ network peak in $S(q)$. This leads to gelation at unphysically high particle densities, thus excluding this scenario [35].

For the systems studied here, gelation is driven by spinodal decomposition into an interconnected colloidrich network, followed by dynamic arrest due to a local glass transition. By contrast, at higher $U$ and lower $\phi$, a state composed of disconnected glassy clusters exists as a precursor to gelation [6]. Such a fluid-cluster state may result from the effects of gravitational sedimentation or charge on the particles [36-38]. Alternatively, these glassy clusters could result from spinodal or binodal decomposition into a phase of disconnected droplets which are then locally arrested by the glass transition, but which do not span space, and hence do not form a gel [12]. The full range of scenarios for the gelation transition depends on $U$, $\xi$, and $\phi$, as well as possible influences of gravity and charge. The results presented here account for one scenario for gelation and serve as a benchmark to compare to other possible mechanisms.

We thank J. Bergenholtz, K. Kroy, M.E. Cates, F. Sciortino, and B. Halperin for helpful discussions. This work was supported by NASA (NAG3-2284), NSF (DMR0243715 and CHE-0134969 [D. R. and K.M.]), the Harvard MRSEC (DMR-0213805), and the Swiss National Science Foundation (H. W. and V. T.).

*Present address: Department of Chemical Engineering, Massachusetts Institute of Technology, Cambridge, MA, 02139, USA.

†resent address: Department of Chemistry, Columbia University, New York, NY, 10027, USA.

[1] S. M. Ilett et al., Phys. Rev. E 51, 1344 (1995).

[2] W. C. K. Poon et al., Phys. Rev. Lett. 83, 1239 (1999).
[3] W. van Megen and P. N. Pusey, Phys. Rev. A 43, 5429 (1991).

[4] K. N. Pham et al., Science 296, 104 (2002).

[5] E. Bartsch, Curr. Opin. Coll. Inter. Sci. 3, 577 (1998).

[6] P. N. Segre et al., Phys. Rev. Lett. 86, 6042 (2001).

[7] W. Gotze, in Liquids, Freezing, and the Glass Transition, edited by J. P. Hansen, D. Levesque, and J. Zinn-Justin (North-Holland, Amsterdam, 1991).

[8] W. van Megen and S. M. Underwood, Phys. Rev. E 49, 4206 (1994).

[9] L. Fabbian et al., Phys. Rev. E 59, R1347 (1999).

[10] M. Carpineti and M. Giglio, Phys. Rev. Lett. 68, 3327 (1992).

[11] J. Bibette et al., Phys. Rev. Lett. 69, 981 (1992).

[12] M.E. Cates et al., J. Phys. Condens. Matter 16, S4861 (2004).

[13] E. Zaccarelli et al., in Proceedings of Unifying Concepts in Granular Materials and Glasses, Capri 2003, edited by A. Coniglio, A. Fierro, H. Hermann, and M. Nicodemi (Elsevier, Amsterdam, 2004).

[14] S. Ramakrishnan et al., J. Chem. Phys. 116, 2201 (2002).

[15] N. A. M. Verhaegh et al., Physica A (Amsterdam) 242, 104 (1997).

[16] K. G. Soga, J. R. Melrose, and R. C. Ball, J. Chem. Phys. 108, 6026 (1998).

[17] V. Prasad et al., Faraday Discuss. 123, 1 (2003).

[18] I. Saika-Voivod et al., Phys. Rev. E 70, 041401 (2004).

[19] E. Zaccarelli et al., Phys. Rev. Lett. 94, 218301 (2005).

[20] S. C. Glotzer et al., Phys. Rev. E 49, 247 (1994).

[21] G. Foffi et al., Phys. Rev. Lett. 94, 078301 (2005).

[22] K. Kroy, M.E. Cates, and W.C.K. Poon, Phys. Rev. Lett. 92, 148302 (2004).

[23] A. Yethiraj and A. van Blaaderen, Nature (London) 421, 513 (2003).

[24] S. Asakura and F. Oosawa, J. Chem. Phys. 22, 1255 (1954).

[25] A. Vrij, Pure Appl. Chem. 48, 471 (1976).

[26] The data were obtained using solutions without added TBAC; this may change the exact values slightly.

[27] J. K. Percus and G. J. Yevick, Phys. Rev. 110, 1 (1958).

[28] A.E. Bailey and D.S. Cannell, Phys. Rev. E 50, 4853 (1994).

[29] E. O. Potma et al., Opt. Lett. 27, 1168 (2002).

[30] J.-X. Cheng and X.S. Xie, J. Phys. Chem. B 108, 827 (2004).

[31] P. N. Pusey and W. van Megen, Physica A (Amsterdam) 157, 705 (1989).

[32] A. M. Puertas, M. Fuchs, and M. E. Cates, J. Phys. Chem. B 109, 6666 (2005).

[33] See EPAPS Document No. E-PRLTAO-95-019549 for a supplementary figure showing the $S(q)$ used as input for the calculations. This document can be reached via a direct link in the online article's HTML reference section or via the EPAPS homepage (http://www.org/pubservs/ epaps.html).

[34] W. Kob, M. Nauroth, and F. Sciortino, J. Non-Cryst. Solids 307-310, 181 (2002).

[35] K. Miyazaki (unpublished).

[36] J. Groenewold and W. K. Kegel, J. Phys. Chem. B 105, 11702 (2001).

[37] H. Sedgwick et al., Eur. Phys. J. E 16, 77 (2005).

[38] F. Sciortino et al., Phys. Rev. Lett. 93, 055701 (2004). 